\title{
Randomized clinical trial of endovenous laser ablation versus direct and indirect radiofrequency ablation for the treatment of great saphenous varicose veins
}

\author{
S. A. S. Hamann ${ }^{1}\left(0\right.$, L. Timmer-de Mik $^{2}$, W. M. Fritschy ${ }^{3}$, G. R. R. Kuiters ${ }^{2}$, T. E. C. Nijsten ${ }^{1}$ \\ and R. R. van den Bos ${ }^{1}$ \\ ${ }^{1}$ Department of Dermatology, Erasmus Medical Centre, Erasmus University Rotterdam, Rotterdam, and Departments of ${ }^{2}$ Dermatology and ${ }^{3}$ Vascular \\ Surgery, Isala, Zwolle, The Netherlands \\ Correspondence to: Dr R. R. van den Bos, Department of Dermatology, Erasmus Medical Centre, Erasmus University Rotterdam, Dr Molewaterplein 40, \\ 3015 GD Rotterdam, the Netherlands (e-mail: r.vandenbos@erasmusmc.nl)
}

\begin{abstract}
Background: The current treatment strategy for many patients with varicose veins is endovenous thermal ablation. The most common forms of this are endovenous laser ablation (EVLA) and radiofrequency ablation (RFA). However, at present there is no clear consensus on which of these treatments is superior. The objective of this study was to compare EVLA with two forms of RFA: direct RFA (dRFA; radiofrequency-induced thermotherapy) and indirect RFA (iRFA; VNUS ClosureFast ${ }^{\mathrm{TM}}$ ).

Methods: Patients with symptomatic great saphenous vein (GSV) incompetence were randomized to receive EVLA, dRFA or iRFA. Patients were followed up at 2 weeks, 6 and 12 months. The primary outcome was GSV occlusion rate. Secondary outcomes included Venous Clinical Severity Score (VCSS), Aberdeen Varicose Vein Questionnaire (AVVQ) score and adverse events.

Results: Some 450 patients received the allocated treatment (EVLA, 148; dRFA, 152; iRFA, 150). The intention-to-treat analysis showed occlusion rates of 75.0 (95 per cent c.i. 68.0 to 82.0), $59.9(52 \cdot 1$ to 67.7) and $81.3(75.1$ to 87.6$)$ per cent respectively after 1 year $(P=0.007$ for EVLA versus $\mathrm{dRFA}, P<0.001$ for dRFA versus $\mathrm{iRFA}, P=\mathbf{0} \cdot 208$ for EVLA versus $\mathrm{iRFA})$. VCSS improved significantly for all treatments with no significant differences between them. AVVQ scores also improved significantly for all treatments, but iRFA had significantly better scores than dRFA at 12 months. Significantly more adverse events were reported after treatment with EVLA (103) than after dRFA (61) and iRFA (65), especially more pain.

Conclusion: Primary GSV occlusion rates were better after iRFA and EVLA than dRFA. All three interventions were effective in improving the clinical severity of varicose veins at 1 year.
\end{abstract}

Paper accepted 3 March 2019

Published online 16 May 2019 in Wiley Online Library (www.bjs.co.uk). DOI: 10.1002/bjs.11187

\section{Introduction}

Varicose veins of the leg, a sign of chronic venous disease, affect around 25-40 per cent of the adult population ${ }^{1-3}$. The aim of treatment is to reduce symptoms, but also to prevent long-term complications of chronic venous insufficiency such as leg ulceration.

The treatment of choice for an incompetent great saphenous vein (GSV) is endovenous thermal ablation ${ }^{4-6}$. There are several ways to ablate the vein, of which laser and radiofrequency are the most frequently used; however, none of the guidelines state which technique is preferred. Although several studies ${ }^{7-11}$ have compared these two treatments, it is still unclear whether endovenous laser ablation (EVLA) is superior to radiofrequency ablation (RFA).
The objective of this study was to compare the efficacy of EVLA and two different forms of RFA: direct RFA (dRFA; radiofrequency-induced thermotherapy) and indirect RFA (iRFA; VNUS ClosureFast ${ }^{\mathrm{TM}}$; VNUS Medical Technologies, St Jose, California, USA). A three-arm, double-blind RCT was undertaken comparing these treatments for GSV incompetence based on occlusion rates after 1 year, with the hypothesis that EVLA would be superior to both dRFA and iRFA.

\section{Methods}

A single-centre, double-blind, three-arm, superiority RCT was carried out at the Phlebology Centre, Isala, Zwolle, between October 2010 and January 2014. The study 
protocol was reviewed and approved by the local medical ethics committee (NL27871.075.10).

Patients were randomized to EVLA, dRFA or iRFA by stratified block randomization using a computer program. In total, three physicians ( 2 dermatologists and 1 vascular surgeon) performed the interventions. Each physician had their own block randomization resulting in an equal distribution of patients among the three treatment groups.

Patients and observers were blinded to the treatment allocation. During treatment, the device was covered such that the patient would not be able to see it. Patients were not allowed to have been treated previously by any of the techniques used here so they would not be able to recognize the sound of the device. The EVLA protocol was used for all patients, which meant they were all asked to wear protective glasses. No information on the technique used was documented in the electronic patient file, only technical information such as length of the treated segment. The physician who evaluated the patient at follow-up visits was different from the one who treated the patient, and was therefore unaware of the technique used. Similarly, the assistants who helped with completion of questionnaires had not been present at the procedure.

Adult patients (aged 18 years and over) with a symptomatic incompetent GSV over a length of at least $15 \mathrm{~cm}$, and with a diameter of $3 \mathrm{~mm}$ or more and no larger than $15 \mathrm{~mm}$, were eligible to participate. GSV incompetence was defined as reflux lasting more than $0.5 \mathrm{~s}$ on colour duplex ultrasound imaging (DUS). Reasons for exclusion were: concomitant ipsilateral small saphenous vein (SSV) incompetence, history of deep vein thrombosis (DVT) in the same leg, active superficial vein thrombosis (SVT) in the GSV, history of surgical or endovenous treatment or ultrasound-guided foam sclerotherapy of the GSV or SSV, pregnancy, BMI $40 \mathrm{~kg} / \mathrm{m}^{2}$ or more, proven peripheral artery occlusive disease (ankle : brachial pressure index below 0.8 ), poor overall health, immobility, and a known coagulation disorder resulting in increased coagulation. For patients with bilateral GSV incompetence, only one leg was included in the study.

In this study the incompetent GSV was treated with either EVLA, dRFA or iRFA.

All endovenous treatments were performed under DUS as described elsewhere ${ }^{12,13}$. For all techniques, the vein was accessed by puncturing the vein with a $16-$ or $18-\mathrm{Fr}$ needle at the lowest point of GSV incompetence, but not more than $10-15 \mathrm{~cm}$ below the knee. After gaining vein access, a guidewire was passed through the needle up to the saphenofemoral junction (SFJ) over which an introducer sheath was passed. The guidewire was removed, and either the laser, dRFA or iRFA fibre/catheter was introduced and positioned approximately $1-2 \mathrm{~cm}$ from the SFJ. Tumescent anaesthetic solution ( $0.5 \mathrm{mg}$ adrenaline (epinephrine), $4.2 \mathrm{mg}$ bicarbonate and $35 \mathrm{ml}$ lidocaine diluted in $500 \mathrm{ml}$ 0.9 per cent saline) was administered under DUS guidance into the saphenous compartment using a mechanical infusion pump.

For EVLA, a 980-nm diode laser (Biolitec ELVeS ${ }^{\mathrm{TM}}$; Biolitec, Vienna, Austria) was used with a bare-tip fibre and power of $10 \mathrm{~W}$. The laser was pulled back continuously, attempting to administer $60 \mathrm{~J} / \mathrm{cm}$. The dRFA catheter (Celon, Teltow, Germany) was also withdrawn in continuous mode and was monitored using the inbuilt acoustic impedance feedback mechanism. If feedback signalled accumulation of coagulum on the applicator tip, the procurve was removed, cleaned and reinserted to continue treatment. The VNUS Closure Fast ${ }^{\mathrm{TM}}$ contained a $7-\mathrm{cm}$ heating element. Two cycles of $20 \mathrm{~s}$ were administered for the first $7 \mathrm{~cm}$ close to the SFJ and only one cycle for the rest of the incompetent GSV. Possible tributaries were not treated, to prevent contamination of the results.

All patients were evaluated by physical examination and DUS at time of inclusion, during treatment, and 2 weeks, 6 and 12 months after treatment.

The primary outcome of the trial was the rate of occlusion after 12 months of follow-up. The following terms were used to evaluate the treated GSV: occlusion, partial occlusion and no occlusion. The GSV was assessed at three points in the leg: proximal (close to the SFJ), medial (halfway along the upper leg) and distal (lowest point of treatment). Occlusion was defined as absence of flow in a non-compressible GSV or an absent GSV at all three measurement points. If there was a segment of compressible GSV with or without flow (antegrade flow or reflux) in one or two of the measurement points, it was rated as partial occlusion. If the treated GSV was still completely open at all three measurement points, it was classified as no occlusion and treatment failure. The analysis comprised evaluation of anatomical success (complete occlusion) versus no anatomical success (partial and no occlusion) as the aim of treatment was to occlude the GSV completely.

Clinical signs of chronic venous disease were assessed by means of the Clinical Etiologic Anatomic Pathophysiologic (CEAP) classification ${ }^{14}$ and the Venous Clinical Severity Score (VCSS) ${ }^{15}$ at baseline, and 6 and 12 months after treatment. The Aberdeen Varicose Vein Questionnaire (AVVQ) $)^{15}$ was used to assess disease-specific quality of life before, and 6 and 12 months after treatment. At all follow-up visits, the occurrence of the following adverse events was registered: pain, haematoma/ecchymosis, DVT, SVT, infection, induration, hyperpigmentation, skin burn, dysaesthesia, nerve damage, pulmonary embolism and 


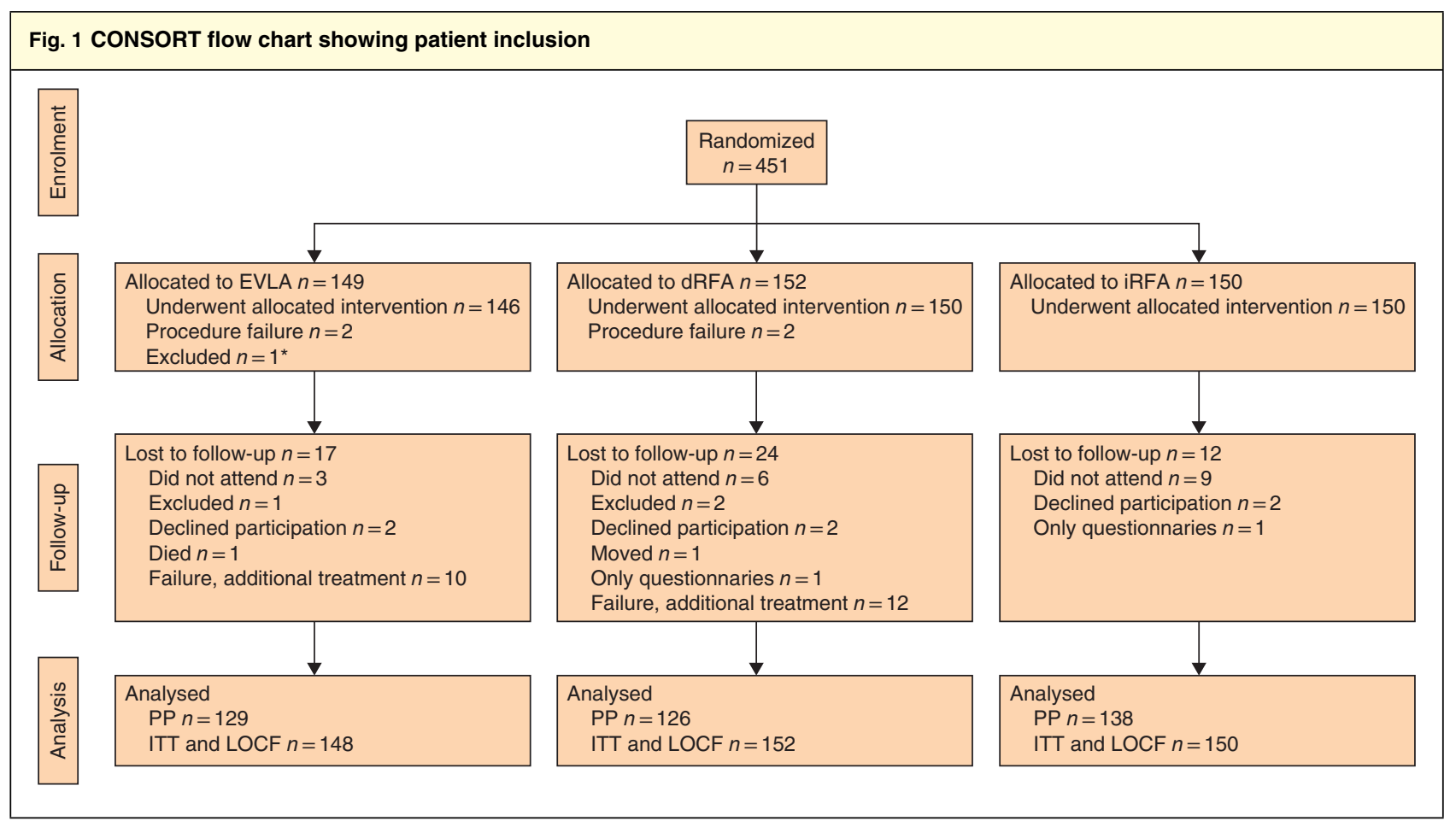

*One patient was excluded as the incompetent vein was actually a tributary and not the great saphenous vein. EVLA, endovenous laser ablation; dRFA, direct radiofrequency ablation; iRFA, indirect radiofrequency ablation; PP, per protocol; ITT, intention to treat; LOCF, last observation carried forward.

'other'. Pain was evaluated using a visual analogue scale (VAS) ranging from 0 (least) to 10 (most) at baseline, during treatment and administration of tumescent anaesthesia, and throughout the first 14 days after treatment. Patients were also asked to register the use of pain medication during those 14 days.

To assess patient satisfaction after treatment, patients were asked if they were satisfied with the treatment they received (satisfied, somewhat satisfied, somewhat unsatisfied, unsatisfied) and if they would choose that treatment again (yes, probably, probably not, no).

\section{Statistical analysis}

The sample size calculation for this superiority design determined that 408 patients (136 per arm) were needed to detect a 10 per cent difference in occlusion rate after 1 year between EVLA and both RFA groups. Possible loss to follow-up of 10 per cent was taken into account, resulting in 450 patients needed for inclusion (150 per treatment arm).

Categorical data were analysed using the $\chi^{2}$ or Fisher's exact test. In the event of a statistically significant difference $(P<0.050)$, the three treatments were compared separately, with $P<0.016$ considered statistically significant. None of the nominal data were distributed normally, so non-parametric tests were used. First, the Kruskal-Wallis test was used to identify possible significant differences between the three treatment groups $(P<0 \cdot 050)$. If the difference was significant, the Mann-Whitney $U$ test was used to analyse the three treatment groups separately, with $P<0.016$ taken to indicate statistical significance. VAS scores during the first 2 weeks after treatment were analysed by means of multivariable analysis.

All analyses were done in SPSS ${ }^{\circledR}$ version 24 (IBM, Armonk, New York, USA) and RStudio ${ }^{\circledR}$ version 1.1.453 (RStudio, Boston, Massachusetts, USA).

\section{Results}

Some 451 patients were randomized in this study, of whom 450 received the allocated treatment (Fig. 1). One patient was excluded because the vein to be treated was actually a tributary rather than the GSV, so this patient did not meet the inclusion criteria. The baseline characteristics for all patients can be found in Table 1. All 450 patients were included in the intention-to treat (ITT) and last-observation-carried-forward (LOCF) analyses. For per-protocol (PP) analyses, a total of 393 patients were 


\begin{tabular}{|c|c|c|c|}
\hline & EVLA $(n=148)$ & dRFA $(n=152)$ & iRFA $(n=150)$ \\
\hline \multicolumn{4}{|l|}{ Patient demographics } \\
\hline Age (years) ${ }^{\star}$ & $51 \cdot 1(13 \cdot 6)$ & $52 \cdot 6(13 \cdot 4)$ & $49 \cdot 3(13 \cdot 9)$ \\
\hline Sex ratio $(F: M)$ & $97: 51$ & $103: 49$ & $105: 45$ \\
\hline \multicolumn{4}{|l|}{ Limb characteristics } \\
\hline Left side & $62(41.9)$ & $72(47 \cdot 4)$ & $71(47 \cdot 3)$ \\
\hline \multicolumn{4}{|l|}{ C class (CEAP classification) } \\
\hline $\mathrm{C} 2$ & $51(34.5)$ & $46(30 \cdot 3)$ & $49(32 \cdot 7)$ \\
\hline $\mathrm{C} 3$ & $76(51.4)$ & $88(57.9)$ & $82(54 \cdot 7)$ \\
\hline $\mathrm{C} 4$ & $14(9.5)$ & $15(9.9)$ & $17(11 \cdot 3)$ \\
\hline C5 & $3(2 \cdot 0)$ & $1(0.7)$ & $2(1 \cdot 3)$ \\
\hline C6 & $3(2 \cdot 0)$ & $2(1 \cdot 3)$ & $0(0)$ \\
\hline Missing & $1(0 \cdot 7)$ & $0(0)$ & $0(0)$ \\
\hline$A V V Q \dagger$ & $9 \cdot 39(6.57-13 \cdot 38)$ & $8 \cdot 14(4 \cdot 36-14 \cdot 26)$ & $7.69(4.82-11.53)$ \\
\hline VCSS $\dagger$ & $4(3-6)$ & $4(3-5)$ & $4(3-5)$ \\
\hline \multicolumn{4}{|c|}{ GSV diameter (supine position) $(\mathrm{mm}) \dagger$} \\
\hline Proximal & $7.8(6.1-9.7)$ & $7 \cdot 6(6 \cdot 2-9 \cdot 3)$ & $7.3(6 \cdot 1-9.5)$ \\
\hline Medial & $5 \cdot 8(4 \cdot 5-7 \cdot 1)$ & $5.5(4.5-7.4)$ & $5.4(4.5-7 \cdot 1)$ \\
\hline Distal & $5 \cdot 4(4 \cdot 3-6 \cdot 9)$ & $5 \cdot 6(4 \cdot 6-7 \cdot 0)$ & $5 \cdot 4(4 \cdot 3-6 \cdot 5)$ \\
\hline \multicolumn{4}{|l|}{ Treatment characteristics } \\
\hline Length of treated segment $(\mathrm{cm})^{\star}$ & $35.5(10 \cdot 7)$ & $37 \cdot 3(10 \cdot 2)$ & $36 \cdot 6(10 \cdot 3)$ \\
\hline Duration of treatment $(\mathrm{min}) \dagger$ & $19 \cdot 5(15 \cdot 5-23 \cdot 1)$ & $16 \cdot 2(12 \cdot 6-19 \cdot 5)$ & $17 \cdot 0(13 \cdot 1-20 \cdot 0)$ \\
\hline Energy used $(\mathrm{J} / \mathrm{cm})^{*}$ & $60 \cdot 1(9 \cdot 85)$ & - & - \\
\hline No. of cycles* & - & $6 \cdot 85(1 \cdot 71)$ & - \\
\hline
\end{tabular}

Values in parentheses are percentages unless indicated otherwise; values are ${ }^{*}$ mean(s.d.) and $\dagger$ median (i.q.r.). EVLA, endovenous laser ablation; dRFA, direct radiofrequency ablation; iRFA, indirect radiofrequency ablation; CEAP, Clinical Etiologic Anatomic Pathophysiologic; AVVQ, Aberdeen Varicose Vein Questionnaire; VCSS, Venous Clinical Severity Score; GSV, great saphenous vein.

analysed; 57 did not complete the 1-year follow-up owing to early failure or loss to follow-up.

Twenty-two patients showed no occlusion at 2 weeks or 6 months (primary treatment failure) and received additional treatment with iRFA, for practical reasons. It was deemed unethical to let these patients complete 1-year follow-up before retreatment, so they received further treatment shortly after initial treatment failure had been established. It was decided to exclude these patients from further follow-up. However, for ITT analysis, the results after retreatment with iRFA would be included in the original treatment group (EVLA or dRFA). For the ITT analysis, these were considered as treatment failures, meaning that their data were imputed as 'no occlusion' for the primary outcome, and no improvement compared with baseline for VCSS and AVVQ scores.

Thirty-one other patients had data missing at 12 months owing to loss to follow-up. For the ITT analysis, these patients were also considered as treatment failures, with missing data imputed as described above. A sensitivity analysis for the primary and secondary (VCSS and AVVQ scores) outcomes was performed, in which missing values were imputed with the last available value, based on the LOCF method. A third analysis using the PP principle was also undertaken.

\section{Anatomical success}

In ITT analysis, GSV occlusion rates were 75.0 (95 per cent c.i. 68.0 to 82.0$), 59.9(52 \cdot 1$ to 67.7$)$ and $81.3(75 \cdot 1$ to 87.6$)$ per cent 1 year after EVLA, dRFA and iRFA respectively (Table 2). Both EVLA $(P=0.007)$ and iRFA $(P<0.001)$ had significantly higher success rates than dRFA. Results for the LOCF and PP analyses were comparable, with success rates of 79.1 and 86.0 per cent respectively for EVLA, 66.4 and 72.2 per cent for dRFA, and 87.3 and 88.4 per cent for iRFA (Table 2). In both these sensitivity analyses, a significant difference was found between DRFA and iRFA $(P<0.001$ for LOCF and $P=0.001$ for PP), and only between EVLA and dRFA in the $\mathrm{PP}$ analysis $(P=0 \cdot 008)$.

At 6-month follow-up, in the ITT analysis, the VCSS had improved to a median of 2 in all groups, which continued to 12 months (Table 3). Results were similar in the LOCF and PP analysis. For all treatment groups and 


\begin{tabular}{|c|c|c|c|c|c|c|c|c|c|}
\hline & \multicolumn{3}{|c|}{ Intention to treat } & \multicolumn{3}{|c|}{ LOCF } & \multicolumn{3}{|c|}{ Per protocol } \\
\hline & $\begin{array}{l}\text { Complete } \\
\text { occlusion }\end{array}$ & $\begin{array}{l}\text { No/partial } \\
\text { occlusion }\end{array}$ & $P^{*}$ & $\begin{array}{l}\text { Complete } \\
\text { occlusion }\end{array}$ & $\begin{array}{l}\text { No/partial } \\
\text { occlusion }\end{array}$ & $P^{*}$ & $\begin{array}{l}\text { Complete } \\
\text { occlusion }\end{array}$ & $\begin{array}{l}\text { No/partial } \\
\text { occlusion }\end{array}$ & $\boldsymbol{P}^{*}$ \\
\hline EVLA & $111(75 \cdot 0)$ & $37(25 \cdot 0)$ & 0.007 versus dRFA & $117(79 \cdot 1)$ & $31(20.9)$ & 0.019 versus $\mathrm{dRFA}$ & $111(86 \cdot 0)$ & $18(14 \cdot 0)$ & 0.008 versus dRFA \\
\hline dRFA & 91 (59.9) & $61(40 \cdot 1)$ & $<0.001$ versus iRFA & $101(66 \cdot 4)$ & $51(33 \cdot 6)$ & $<0.001$ versus iRFA & $91(72 \cdot 2)$ & $35(27 \cdot 8)$ & 0.001 versus iRFA \\
\hline iRFA & $122(81 \cdot 3)$ & $28(18.7)$ & 0.208 versus EVLA & $131(87 \cdot 3)$ & $19(12 \cdot 7)$ & 0.063 versus EVLA & $122(88.4)$ & $16(11 \cdot 6)$ & 0.586 versus EVLA \\
\hline
\end{tabular}

Values in parentheses are percentages. LOCF, last observation carried forward; EVLA, endovenous laser ablation; dRFA, direct radiofrequency ablation; iRFA, indirect radiofrequency ablation. ${ }^{*} \chi^{2}$ test.

\begin{tabular}{|c|c|c|c|}
\hline & EVLA & dRFA & iRFA \\
\hline \multicolumn{4}{|l|}{ vcss } \\
\hline Baseline & $4(3-6)$ & $4(3-5)$ & $4(3-5)$ \\
\hline 6 months & $2(1-3)$ & $2(1 \cdot 25-3)$ & $2(1-3)$ \\
\hline 12 months & $2(1-3)$ & $2(1-4)$ & $2(1-3)$ \\
\hline \multicolumn{4}{|l|}{ AVVQ score } \\
\hline Baseline & $9.39(6.57-13.38)$ & $8 \cdot 14(4 \cdot 36-14 \cdot 26)$ & $7.69(4.82-11.53)$ \\
\hline 6 months & $2 \cdot 64(0.69-6.69)$ & $2.46(0.69-7.74)$ & $1.74(0.65-4.63)$ \\
\hline 12 months & $2.57(0.53-7.36)$ & $3.74(0.86-9.36)$ & $1.92(0.34-5.74)$ \\
\hline
\end{tabular}

Values are median (i.q.r.). EVLA, endovenous laser ablation; dRFA, direct radiofrequency ablation; iRFA, indirect radiofrequency ablation; VCSS, Venous Clinical Severity Score; AVVQ, Aberdeen Varicose Vein Questionnaire.

sensitivity analyses this improvement was statistically significant $(P<0 \cdot 001)$, but no significant differences were found between the three treatments.

Median AVVQ scores at baseline ranged from 7.69 to 9.39 and had improved significantly after 6 months for all three treatments $(P<0 \cdot 001)$ (Table 3). This improvement was still present after 12 months. For this analysis, a significant difference was found between dRFA and iRFA $(P=0.009)$. The LOCF analysis showed slightly better results, with median scores of 2.33 (i.q.r. $0.52-5.75$ ) for EVLA, $3.21(0.86-9.33)$ for dRFA and $1.57(0.34-4.94)$ for iRFA after 12 months. Again, a significant difference was found between dRFA and iRFA $(P=0.006)$, but there was no significant difference for EVLA versus dRFA or iRFA. Median scores in the PP analysis were $2.28(0.52-5.46)$ for EVLA, $2.71(0.60-7.06)$ for dRFA and $1.57(0.3-5.08)$ for iRFA after 12 months, with no significant differences between the three treatment groups.

\section{Adverse events}

The occurrence of adverse events was registered at all follow-up visits. No severe adverse events such as DVT or pulmonary embolism were reported during the study. One

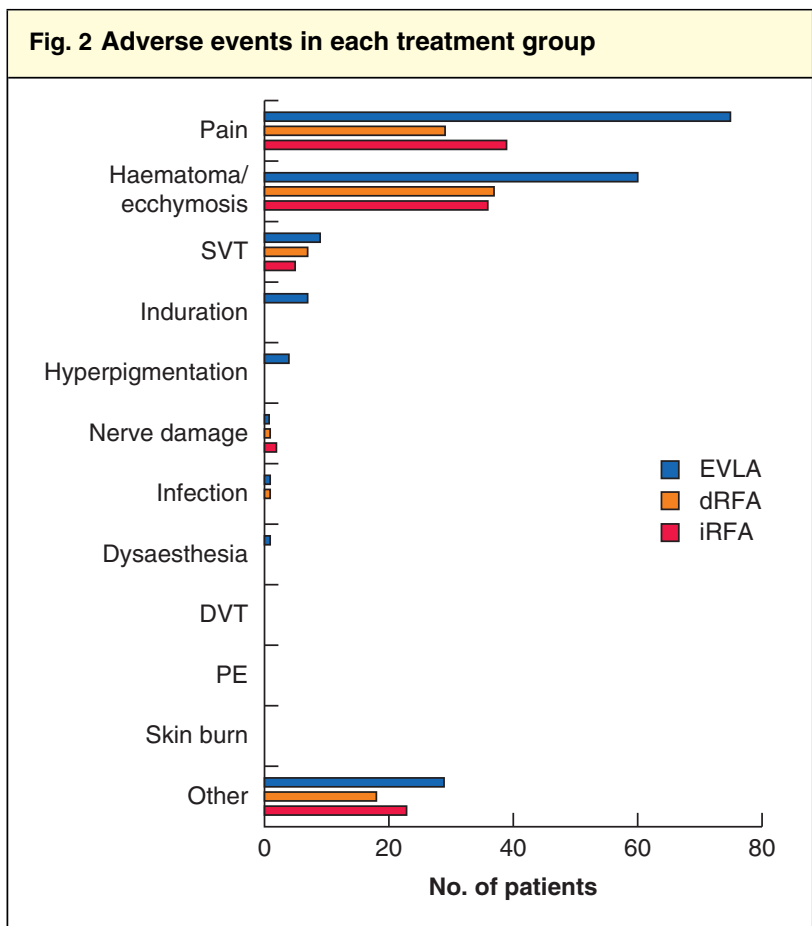

EVLA, endovenous laser ablation; dRFA, direct radiofrequency ablation; iRFA, indirect radiofrequency ablation; SVT, superficial vein thrombosis; DVT, deep vein thrombosis; PE, pulmonary embolism.

or more adverse events in the first 2 weeks after treatment were reported by 103 of 146 patients ( 70.5 per cent) in the EVLA group, 61 of 146 (41.8 per cent) in the dRFA group and 65 of 150 in the iRFA group ( 43.3 per cent). The differences between EVLA and DRFA, and EVLA and iRFA were statistically significant $(P<0.001)($ Fig. 2$)$. Pain was the most frequently reported adverse event in all three groups, but significantly more after EVLA than after dRFA and iRFA (both $P=0 \cdot 007$ ).

\section{Pain and patient satisfaction}

During administration of tumescent anaesthetic, VAS scores were similar in all treatment groups, with median 
values of $2 \cdot 3$ (i.q.r. $0 \cdot 8-3 \cdot 7)$ for EVLA, $2.7(1.4-2 \cdot 9)$ for dRFA and $2.7(1.4-4.4)$ for iRFA $(P=0 \cdot 115)$. Median pain scores during treatment were $0 \cdot 2(0-0 \cdot 8), 0 \cdot 2(0-1 \cdot 1)$ and $0.6(0 \cdot 1-2 \cdot 6)$ respectively. Scores were significantly higher for iRFA than for either EVLA $(P<0.001)$ or dRFA $(P=0.001)$.

VAS scores reported by patients during the first 14 days after treatment showed different patterns in the three treatment groups. Patients treated with EVLA reported higher pain scores directly after treatment than those in the other groups, with a mean(s.d.) of $2 \cdot 2(1 \cdot 9)$, but this improved over time with an intercept of $0 \cdot 1$. After dRFA, the pain scores were initially low, with a mean of $0 \cdot 8(0 \cdot 9)$, and decreased slightly over time with an intercept of 0.04 . For patients treated with iRFA, the pain scores were initially low (mean VAS $0 \cdot 4(0 \cdot 0)$ ), but increased slightly over time with an intercept of $0 \cdot 1$. Comparing these patterns over time, there was no difference between EVLA and dRFA, but EVLA and iRFA differed significantly $(P=0.012)$.

However, significantly more patients treated with EVLA used pain medication ( 95 of $143,66.4$ per cent) than patients treated with dRFA (57 of $149,38.3$ per cent) and iRFA (63 of $148,42.6$ per cent) (both $P<0.001$ ).

At the first follow-up visit 2 weeks after treatment, patients were asked if they were satisfied with the treatment they received and would choose the same treatment again. For EVLA, 136 of 145 (93.8 per cent) were satisfied or somewhat satisfied with their treatment, compared with 148 of 150 (98.7 per cent) for dRFA and 149 of 150 (99.3 per cent) for iRFA. Only the difference between EVLA and iRFA was statistically significant $(P=0.009)$. Some $90 \cdot 3$ per cent of the patients treated with EVLA would choose the same treatment again, compared with 97.3 and 98.0 per cent of those who had dRFA and iRFA respectively $(P=0.014$ and $P=0.005)$.

\section{Discussion}

In this three-arm study, both EVLA and iRFA were significantly more effective than dRFA in treating GSV incompetence 1 year after treatment. Patients treated with EVLA reported more adverse events, specifically pain, and used more pain medication after treatment than those who had dRFA or iRFA. VAS scores during iRFA were significantly higher than those during EVLA and dRFA, but the difference of 0.4 was not considered clinically relevant. VAS pain scores during the first 2 weeks after treatment were higher after EVLA than either dRFA or iRFA. Curiously, the pain scores after iRFA seemed to increase slightly during this interval. However, the pain scores varied widely and too few patients registered pain scores for the full
14 days to draw reliable conclusions based on this analysis. VCSS improved significantly in all groups, with no significant differences between the three treatments after 1 year. AVVQ scores also improved significantly after all three treatments; however, both the ITT and LOCF analysis showed significantly better AVVQ scores after iRFA than dRFA.

Several non-blinded studies have compared the efficacy of EVLA, iRFA and dRFA, with inconclusive results. One meta-analysis ${ }^{7}$ and a systematic review ${ }^{16}$ both suggested higher occlusion rates for EVLA than for iRFA, with less pain after iRFA. The results of a four-arm $\mathrm{RCT}^{17} \mathrm{com}-$ paring EVLA, iRFA, ultrasound-guided foam sclerotherapy and conventional surgery were similar to those of the present study, with comparable occlusion rates for EVLA and iRFA, but also more pain after EVLA.

A plausible explanation for the large number of failures in the dRFA group could be the fact that this technique requires steps that are different from those in both EVLA and iRFA, which are relatively similar in how they are conducted. With the dRFA technique, it is often necessary to remove the wire and clean it one or more times, after which treatment is continued. This probably makes treatment with dRFA more prone to error and it requires more experience. One of the physicians had less experience with dRFA before the study than the other two. This could explain why this physician had around 30 failures in the dRFA group, whereas the other two had around ten failures each.

Patients who had EVLA were less satisfied with their treatment, and fewer patients would choose the treatment again, compared with those treated with dRFA and iRFA. This may be because the patients who had EVLA experienced more adverse events, and specifically more pain, than patients in the RFA groups.

This study has some limitations. Fifty-seven of the 450 patients did not complete the 1-year follow-up, 22 owing to early treatment failure resulting in exclusion from further follow-up. It would have been interesting to determine the influence of early failure on outcomes such as VCSS and AVVQ scores, but it was deemed unethical to allow these patients to complete follow-up before retreatment. These patients were excluded from follow-up, but their data were included in the ITT and LOCF analyses. It should also be noted that a 980-nm fibre was used for EVLA, whereas $1470 \mathrm{~nm}$ is currently the preferred wavelength as it might be associated with less postoperative pain ${ }^{18}$.

\section{Acknowledgements}

This study was not preregistered in an independent registry. 
Disclosure: The authors declare no conflict of interest.

\section{References}

1 Evans CJ, Fowkes FG, Ruckley CV, Lee AJ. Prevalence of varicose veins and chronic venous insufficiency in men and women in the general population: Edinburgh Vein Study. 7 Epidemiol Community Health 1999; 53: 149-153.

2 Rabe E, Berboth G, Pannier F. [Epidemiology of chronic venous diseases.] Wien Med Wochenscbr 2016; 166: 260-263.

3 Rabe E, Guex JJ, Puskas A, Scuderi A, Fernandez Quesada F; VCP Coordinators. Epidemiology of chronic venous disorders in geographically diverse populations: results from the Vein Consult Program. Int Angiol 2012; 31: $105-115$.

4 Nicolaides A, Kakkos S, Eklof B, Perrin M, Nelzen O, Neglen $\mathrm{P}$ et al. Management of chronic venous disorders of the lower limbs - guidelines according to scientific evidence. Int Angiol 2014; 33: 87-208.

5 Wittens C, Davies AH, Bækgaard N, Broholm R, Cavezzi A, Chastanet $\mathrm{S}$ et al. Editor's choice - management of chronic venous disease: Clinical Practice Guidelines of the European Society for Vascular Surgery (ESVS). Eur 7 Vasc Endovasc Surg 2015; 49: 678-737.

6 National Institute for Health and Care Excellence (NICE). Varicose veins: Diagnosis and Management; 2013. https://www .nice.org.uk/guidance/cg168 [accessed 18 February 2019].

7 van den Bos R, Arends L, Kockaert M, Neumann M, Nijsten T. Endovenous therapies of lower extremity varicosities: a meta-analysis. 7 Vasc Surg 2009; 49: 230-239.

8 Bozoglan O, Mese B, Eroglu E, Ekerbiçer HC, Yasim A. Comparison of endovenous laser and radiofrequency ablation in treating varices in the same patient. 7 Lasers Med Sci 2017; 8: 13-16.

9 Mese B, Bozoglan O, Eroglu E, Erdem K, Acipayam M, Ekerbicer HC et al. A comparison of 1470-nm endovenous laser ablation and radiofrequency ablation in the treatment of great saphenous veins $10 \mathrm{~mm}$ or more in size. Ann Vasc Surg 2015; 29: 1368-1372.
10 Lawaetz M, Serup J, Lawaetz B, Bjoern L, Blemings A, Eklof $\mathrm{B}$ et al. Comparison of endovenous ablation techniques, foam sclerotherapy and surgical stripping for great saphenous varicose veins. Extended 5-year follow-up of a RCT. Int Angiol 2017; 36: 281-288.

11 Woźniak W, Mlosek RK, Ciostek P. Complications and failure of endovenous laser ablation and radiofrequency ablation procedures in patients with lower extremity varicose veins in a 5-year follow-up. Vasc Endovascular Surg 2016; 50: 475-483.

12 Nijsten T, van den Bos RR, Goldman MP, Kockaert MA, Proebstle TM, Rabe $\mathrm{E}$ et al. Minimally invasive techniques in the treatment of saphenous varicose veins. $7 \mathrm{Am}$ Acad Dermatol 2009; 60: 110-119.

13 Goodyear SJ, Nyamekye IK. Radiofrequency ablation of varicose veins: best practice techniques and evidence. Phlebology 2015; 30(Suppl): 9-17.

14 Eklöf B, Rutherford RB, Bergan JJ, Carpentier PH, Gloviczki P, Kistner RL et al.; American Venous Forum International Ad Hoc Committee for Revision of the CEAP Classification. Revision of the CEAP classification for chronic venous disorders: consensus statement. 7 Vasc Surg 2004; 40: 1248-1252.

15 Vasquez MA, Munschauer CE. Venous Clinical Severity Score and quality-of-life assessment tools: application to vein practice. Phlebology 2008; 23: 259-275.

16 Ahadiat O, Higgins S, Ly A, Nazemi A, Wysong A. Review of endovenous thermal ablation of the great saphenous vein: endovenous laser therapy versus radiofrequency ablation. Dermatol Surg 2018; 44: 679-688.

17 Rasmussen LH, Lawaetz M, Bjoern L, Vennits B, Blemings A, Eklof B. Randomized clinical trial comparing endovenous laser ablation, radiofrequency ablation, foam sclerotherapy and surgical stripping for great saphenous varicose veins. Br 7 Surg 2011; 98: 1079-1087.

18 Malskat WS, Giang J, De Maeseneer MG, Nijsten TE, van den Bos RR. Randomized clinical trial of 940- versus 1470-nm endovenous laser ablation for great saphenous vein incompetence. Br F Surg 2016; 103: 192-198. 